\title{
Development of the first commercial real-time PCR assay for the detection of Mucorales species (MucorGenius)
}

\author{
Giel Gaajetaan ${ }^{1}$, Dennis van Tegelen ${ }^{1}$, Tim Kampermann ${ }^{1}$, Gijs Dingemans ${ }^{1}$
}

1. PathoNostics B.V., Maastricht, The Netherlands

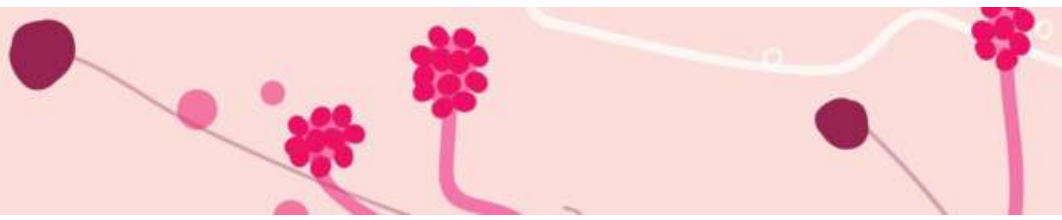

\section{Introduction}

Mucormycosis is a rare invasive fungal infection caused by Mucorales species and generally affects severely immunocompromised individuals. Mucormycosis is associated with a high mortality rate and prevalence is underestimated due to the limited diagnostic options. Correct and fast identification of mucorales infection is essential to start appropriate antifungal therapy.

Due to this limitation in current diagnostics, PathoNostics developed a real-time PCR for the detection of the clinical most relevant Mucorales species. The goal of this study was to determine the analytical specificity, inclusivity and sensitivity. The performance of the MucoGenius PCR was also examined on a set of ring trial samples.

\section{Materials \& Methods}

The real-time PCR assay (MucorGenius, PathoNostics) enables detection of the $28 \mathrm{~S}$ multicopy gene in Mucorales species together with an internal control.

In order to determine the analytical sensitivity, serial DNA dilutions of 7 reference strains including $R$. oryzae, $M$. racemosus, L. corymbifera, $R$. microsporus, $C$. bertholletiae, $R$. pusillus and $R$. miehei were quantified using droplet digital PCR (ddPCR).

Quality assessment was performed using the ring trial of the Fungal PCR Initiative (FPCRI) for Mucorales species.

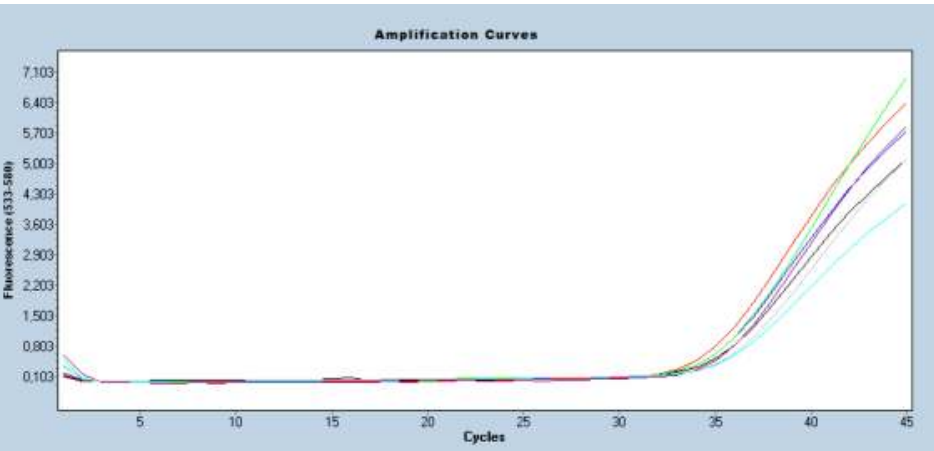

Figure 1. Mucorales amplification curves of the highest, detectable DNA dilutions. Detection of $L$. corymbifera, $R$. oryzae, $M$.racemosus, $R$. micropsorus, C. bertholletiae, $R$. pusillus and $R$. miehei DNA.

\section{Results}

The MucorGenius PCR was able to specifically detect all tested Mucorales species. No cross-reactivity was observed with other fungi, yeast or bacterial strains commonly present in the respiratory tract (data not shown).

The analytical sensitivity was determined as the highest dilution of Mucorales DNA which could be detected with the MucorGenius and is quantified with ddPCR (figure 1). All MucorGenius targets were detected at 1-2 copies/ $\mu$ l of extracted DNA which corresponds with a Ct-value of 35/36 on the LC480 II real-time PCR (table 1).

The MucorGenius PCR identified Mucorales in 5 out of 6 FPCRI samples, which was in agreement with the FPCRI data (table 2).

\section{Conclusion}

* The MucorGenius real-time PCR from PathoNostics can specifically detect the clinically most relevant Mucorales species in low concentrations.

* The MucorGenius PCR had a 100\% score with the FPCRI panel.

* Further validation on clinical samples is ongoing.

\begin{tabular}{|l|l|l|l|}
\hline Sample nr. & Inoculated DNA & MucorGenius & Ct-value \\
\hline S1 & R. pusillus & + & 34 \\
\hline S2 & None & - & Negative \\
\hline S3 & R. oryzae & + & 32 \\
\hline S4 & C. bertholletiae & + & 33 \\
\hline S5 & L. corymbifera & + & 34 \\
\hline
\end{tabular}

Table 2. FPCRI Mucorales panel results.

\begin{tabular}{|c|c|c|}
\hline \multicolumn{2}{|c|}{ ddPCR results analytical sensitivity MucorGenius } \\
\hline Target & Conc. (copies/ $\mu \mathrm{l})$ & Ct-value \\
\hline L. corymbifera & 1 & 35 \\
\hline R. oryzae & 1 & 35 \\
\hline M. racemosus & 1 & 35 \\
\hline R. microsporus & 1 & 36 \\
\hline C. bertholletiae & 2 & 35 \\
\hline R. pusillus & 2 & 35 \\
\hline R. miehei & 2 & 36 \\
\hline
\end{tabular}

Table 1. Analytical sensitivity of the MucorGenius PCR for all tested Mucorales species. Ct-values were determined on the LC480 II PCR. 